Pre-print

\title{
Evaluating the impact of an integrated urban design of transport infrastructure and public space on human behavior and environmental quality: A case study in Beijing
}

Liu Yang, Koen H. van Dam, Bani Anvari and Audrey de Nazelle

\begin{abstract}
Urban transport infrastructure can result in the physical, psychological and environmental separation of neighborhoods, public spaces and pedestrian network, leading to negative impacts on citizens' daily commutes, social activities and the quality of ecosystem. An integrated design of transport infrastructure and public space is beneficial for mediating these negative impacts. In this paper, we propose an integrated methodology, which combines urban design, computational scenario evaluation and decision-making processes, based on a conceptual model of human-ecology needs driven planning. To evaluate the impacts of road network and public space design on individual outdoor activities, travel behavior and air pollution, an agent-based model is demonstrated. This model is then applied to a case study in Beijing, leading to hourly traffic volume maps and car-related air pollution heat maps of a baseline road network-public space design.
\end{abstract}

\section{Keywords}

urban transport infrastructure, human behavior, public space, agent-based modeling, policy-making support, environmental evaluation, air pollution simulation

Liu Yang $(\bowtie)$

Center of Architecture Research and Design, University of Chinese Academy of Sciences, Beijing 100190, China e-mail: yangliu113@mails.ucas.ac.cn

Koen H. van Dam

Department of Chemical Engineering, Imperial College London, London SW7 2AZ, UK

e-mail: k.van-dam@imperial.ac.uk

Bani Anvari

Centre for Transport Studies, University College London,London WC1E 6BT, UK

e-mail: b.anvari@ucl.ac.uk

Audrey de Nazelle

Centre for Environmental Policy, Imperial College London, London SW7 2AZ, UK

e-mail: anazelle@imperial.ac.uk 


\section{Introduction}

After the Industrial Revolution, patterns of cities changed prominently as a result of urban growth. Urban transport infrastructure (e.g. road, railway and light rail) has taken a prominent role in shaping the urban fabric. In developing countries, governments have increasingly invested in infrastructure development to meet the need of rapid urbanization. However, because motorized transportation development was prioritized over public realm design in many cities, the transport infrastructure is sometimes represented as a linear separating component that cuts the urban fabric, especially the city centers, into fractured, inaccessible and unfriendly pieces of urban spaces. Such spaces, named by Carmona (2003) as Space Leftover After Planning, have low visual aesthetic quality, minimal public urban facilities and no meaningful purposes. Therefore, they are less used by citizens for doing physical activity (i.e. recreational activity or active travel), which is a significant element of improving public health. Simultaneously, active travel facilities such as pavements and bicycle lanes around such linear transport components are typically disconnected, leading to inconveniences to walk and cycle in city centers.

Furthermore, the construction of transport infrastructures could cut the urban ecosystem into separate tracts of green spaces, thereby influencing the system's ecological resilience and leaving it vulnerable to shifts in climate change (Alberti and Marzluff 2004). The unconnected blocks in city centers raise individual's dependence on motorized transportation, resulting in a great amount of air pollution. Bad air quality in turn impacts on human behavior and results in a reduction of active travel and physical activity in outdoor urban spaces.

It has been widely accepted that an integration of the urban transport infrastructure and land-use is an essential precondition for sustainable development (Varnelis 2008). Some others went a step further demonstrating that transport infrastructures and public spaces should be designed as a holistic system (Ravazzoli and Torricelli 2017). In this respect, this paper focuses on the impacts of an integrated transport and public space design on human behavior and the ecosystem. Here, the public space refers to streets, public open spaces (e.g. parks) and public urban facilities (e.g. markets) (UN-Habitat 2015). Initially, this paper aims at exploring the relationship between transport-human behavior-ecosystem, leading to a conceptual model. Then we attempt to propose a methodology integrating urban design, 
computational evaluation of design scenarios and decision-making support. Moreover, this research aims to show how computer modeling can be used to visualize the impacts of road network and public space designs on outdoor activities, travel behavior and car-related air pollution. Finally, the proposed simulation model will be tested on a case study in Beijing in which real-time data validation and air pollution calculation are conducted.

\section{Literature Review}

Much research in recent years has focused on the reciprocal relationship between public health and active travel policy, which encourages walking and cycling. In the relation loop, transport and planning policies influence human behavior (in terms of the travel mode choice, route choice and physical activity) and environmental quality, which jointly influence human exposure to the environment (e.g. the exposure to air pollution, heat and traffic injuries) (De Nazelle et al. 2011).

In the domain of urban planning, a group of urban theorists inspired by nature and developed a concept of Bio-inspired Urbanism, which regards a city as an evolving organism (Geddes 1915). At the present time, the self-organization in biological systems has been introduced into the study of urban systems, leading to widespread discussions and practices in exploring the analogs between the built environment or social unit and ecological processes. According to the urban dynamics theory (Forrester 1969), others have treated a city as a complex system consisting of interconnected sub-systems-- two of which are transport infrastructure and public space systems. On the other hand, Needs Theory (e.g. Mallmann 1980) has been adopted to urban plan in order to achieve a bottom-up planning process. Jackson et al. (2004) argued that a human needs-based construction offers the possibility of sustainable development and "good life" in present-day society.

In terms of the urban design methodology, the traditional esthetic and empirical way of urban design has been criticized for a lack of quantitative evaluation. In this respect, mathematical and computational modeling tools have been gradually combined with the traditional linear process of "survey-analysis-plan" to achieve an integrated design-evaluation process, which involves planners, modelers and other designers together in a design process (Batty et al. 2012; Gan 2014). For instance, the transport and land-use sub-systems have been holistically planned and assessed by planners using computer 
models such as MATSim (Horni et al. 2016) and Integrated Transport-Land Use models (e.g. Zhao et al. 2013), while little work has been done to build up integrated simulation models for transport and public space systems.

One of the initial policy-making models was described by Axelrod (1976), focusing on the causal analysis used by an individual to evaluate complex policy alternatives. The multiplicity of dimensions in a coupled transport-public space system imposes difficulties to determining clear causations by one individual, thus an integrated analysis approach involving scientists, policy-makers and the public is needed. Moreover, researchers have pointed out that such analysis approach should be designed as an internal part of the decision-making process since stakeholders can judge the feasibility of an approach by learning about a system and exploring possible outcomes of different scenarios (Zellner 2008). Since major infrastructure and policy decisions need to be based on sound evidence, computer modeling (with advantages of real-time data validation and prediction) is an efficient way to facilitate the analysis and evaluation of different design scenarios which would then supplement decision-making. Among others, Agent-Based Modeling (ABM) has been markedly applied to provide qualitative insights and quantitative results for the cooperation among decision makers (Axelrod 1997).

\section{Methodology}

To explore the impact of transport infrastructure planning on human behavior and environmental quality, this paper initially analyzes the interactive mechanism underlying transport infrastructure, human behavior and the ecosystem. Needs theories and bio-inspired urbanism theories are adapted to create a conceptual model, presented in Sec. 4.

This paper then apply the conceptual model to designing and evaluating an integrated transport-public space scenario, resulting in an integrated methodology for human-needs driven design and computational evaluation. This methodology, shown in Sec. 5, is in line with the design-evaluation process demonstrated by van Dam et al. (2014) in which an initial design was evaluated using a simulation. However, novel steps are made by integrating the traditional linear process of urban design, big data input and decision-making process. Furthermore, metrics for evaluating the impacts of transport 
infrastructure and public space designs on human behavior and environmental quality are provided by referring to (De Nazelle et al. 2009).

In the part of computational evaluation, ABM is chosen because it provides a possibility of simulating human's needs and behavior by generating a synthetic population from the given statistics, by which the emergence of social behavior could be witnessed. A case study using this computer model is demonstrated in Sec.6. Aiming at visualizing the traveling of agents between homes, workspaces and public spaces, we adopt and revise the Smart-City Model proposed in (Bustos-Turu et al. under review; van Dam et al. 2017), which has separate layers of road network, land-use and agents.

Initially, a synthetic population as a representative of the entire population of a research field is generated. Using socio-demographic data (the population density, household size, the ratio of worker and non-worker, and car ownership) and geographical data (public spaces, other land-uses and activity distribution), agents are generated randomly based on the number of people living in each area. Then, activity patterns (i.e. the time-specific sequence of activities throughout a weekday or weekend) are designated for agents which are different for included agent types (e.g. workers, residents who are not economically active, and visitors). In order to categorize human activities according to human needs, five types of activities are elicited: residential activity, industrial activity, commercial activity, cultural activity, and leisure activity. In the land-use layer, the residential activity is given to residential areas, the industrial activity is set to workplaces, and the other three activities are assigned to public spaces. In addition, motorized and active travel road networks are provided to agents for traveling to different locations, during which period they follow a shortest path choice algorithm-- Dijkstra's algorithm (Skiena 1998) - to choose their routes. The model are run for a baseline scenario.

To calculate air pollution emitted by vehicles, we track car agents, which are created according to the car-ownership of the total agents and plan their routes only on the motorized road network. Subsequently, emission on each road is calculated as follows: the traffic volume (the number of agents traveled) on each road segment is recorded hourly, after which the volume is compared with real-time traffic data. By using the software of COPERT, the traffic volume is transformed to the emission rate of $\mathrm{NO}_{2} \mu \mathrm{g} /(\mathrm{s} * \mathrm{~m})$, which is visualized in an air pollution heat map. 


\section{A conceptual model}

DNA, as a molecule of the self-organized biological system, is similar to the urban system. Inspired by the double helix structure of DNA, a conceptual model of the urban infrastructure plan was found (see Fig. 1). Corresponding to the two helical chains in DNA, human and ecology needs form the backbone of an urban system, since human demands drive the development and functioning of a city, and the ecology quality restricts urban growth. Here, human needs include personal, social and environmental aspects, and ecology needs refer to biodiversity, networked green and water system. Depending on the human-ecology needs strands, urban transport infrastructure, as well as the other five types of urban infrastructure systems, should be constructed to meet and connect the two needs, functioning in the same way as the base pairs in DNA. This prototype could support policy makers by considering the human-ecology needs as vital intervening variables that shape and constrain their actions; moreover, the needs strands are beneficial for establishing boundaries within which the land-use and transport planning, urban design proposals, public health initiatives and environmental policies are made.

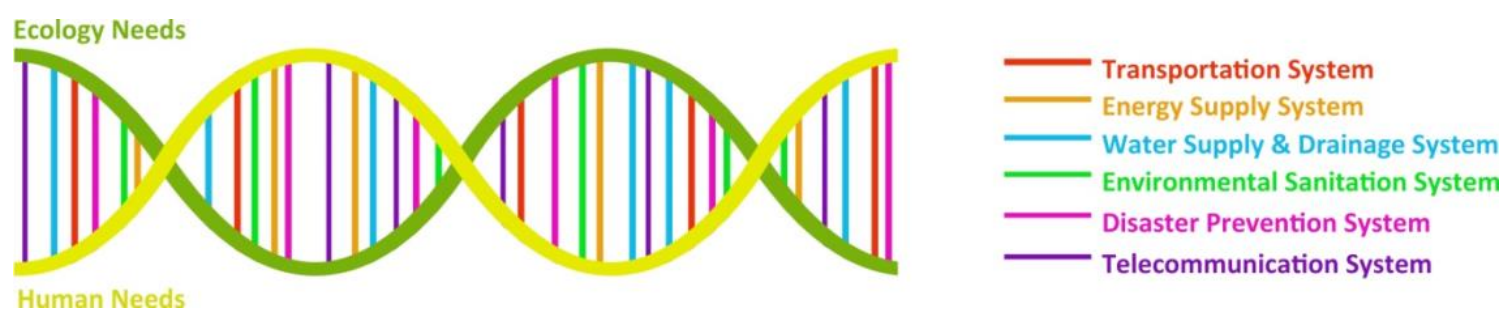

Fig. 1 The DNA form of urban infrastructure plan.

\section{An integrated methodology}

To achieve an integrated methodology supporting both a human-ecology needs driven transport infrastructure design and a quantitative evaluation, the traditional design process of "survey-analysis-plan" could be combined with computer simulation. A simulation could be utilized to analyze the mechanism of a given transport infrastructure and public space system aided by big data. Fig. 2 shows a promising framework, which incorporates human needs in the steps of survey, system simulation (e.g. individual travel data input), evaluation (by assessing the metrics of physical activity) and 
decision-making. Ecology needs are incorporated in the step of problem analysis and evaluation (by assessing the metrics of air quality).

In the loop of scenario design, after the physical transport and public space planning, scenarios are set into an agent-based model to simulate their impacts on human behavior (i.e. physical activity pattern, travel mode choice, route choice and destination choice) and air pollution. Then comparisons with real-time on-road traffic data are carried out in order to validate the model. Moreover, this model could be used to monitor the population's exposure to air pollution, which is a vital metric of evaluating transport scenarios. In this integrated model, public participation appears in the stage of urban context survey, the stage of decision-making (i.e. the public is involved by observing the modeling process and providing suggestions) and finally in the long-term monitoring after implementing one of the transport plans. It is worth noting that this method is more than a linear process, but an iterative progress that feedback getting from monitoring a designed transport and public space system in one, five, or ten years will yield redesigns of the system.

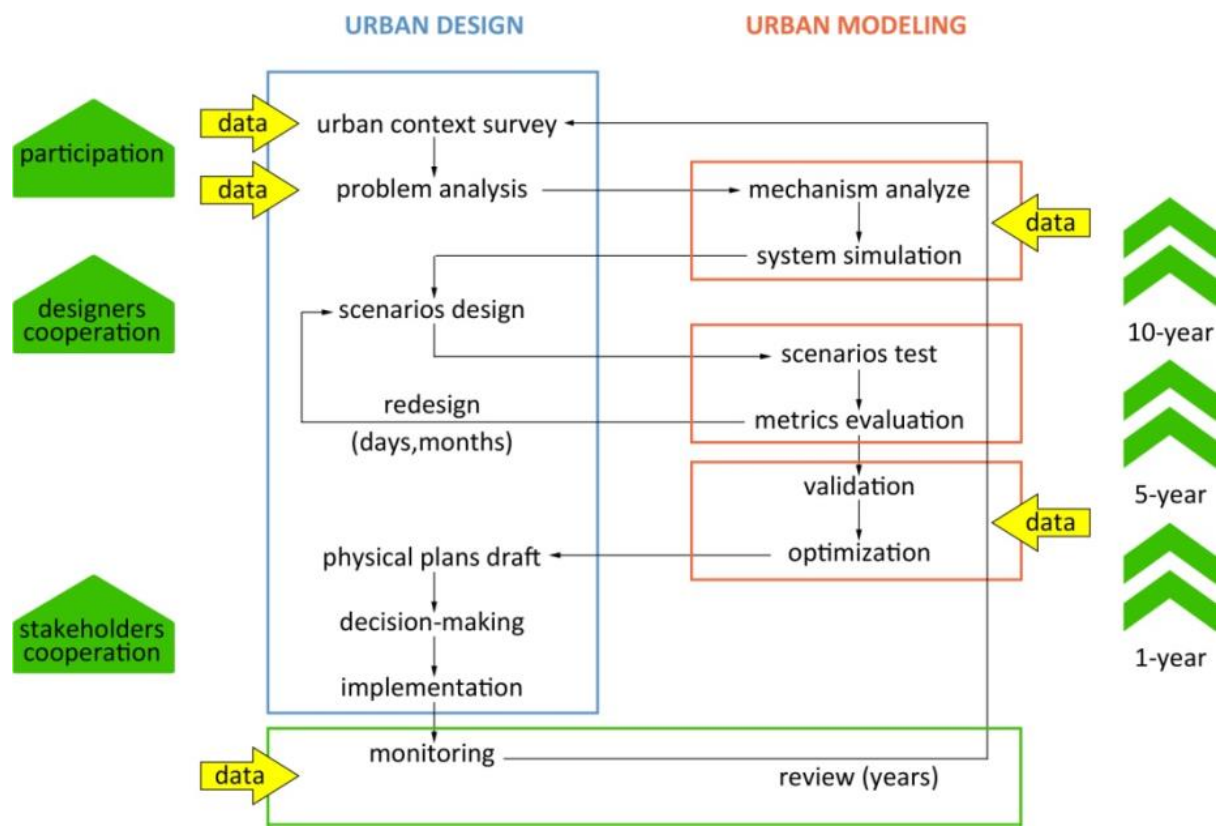

Fig. 2 A methodology integrating urban design and modeling to evaluate design scenarios, with giving insights in key performance indicators for policy-making support.

\section{Case study and results}

Beijing, the capital of China, is one of the world's most global, dense and ancient cities. The Beijing-Zhangjiakou 
railway, which was the very first urban rail constructed in Beijing, cuts through the city center. In order to upgrade the railway for the use of Beijing 2022 Winter Olympic Games, the out-of-date rail track was demolished and will be replaced by a high-speed rail underground. Fig. 3 shows the research field chosen for this study, a 10-kilometer section of the Beijing-Zhangjiakou railway, while the status quo of disconnected urban spaces lying around this section is presented in Fig. 4. Facing a detrimental problem of heavy air pollution, indicators of environmental impacts and human behavior changes have been gradually taken into account when redesigning transport infrastructures and public spaces in Beijing. To compare various design proposals using metrics such as environmental quality, physical activity and human exposure could help policy-making. In this paper, we begin by applying an ABM to simulate the baseline scenario of the transport infrastructure and public space system, based on which alternative transport and public space designs can be tested and compared in future work.
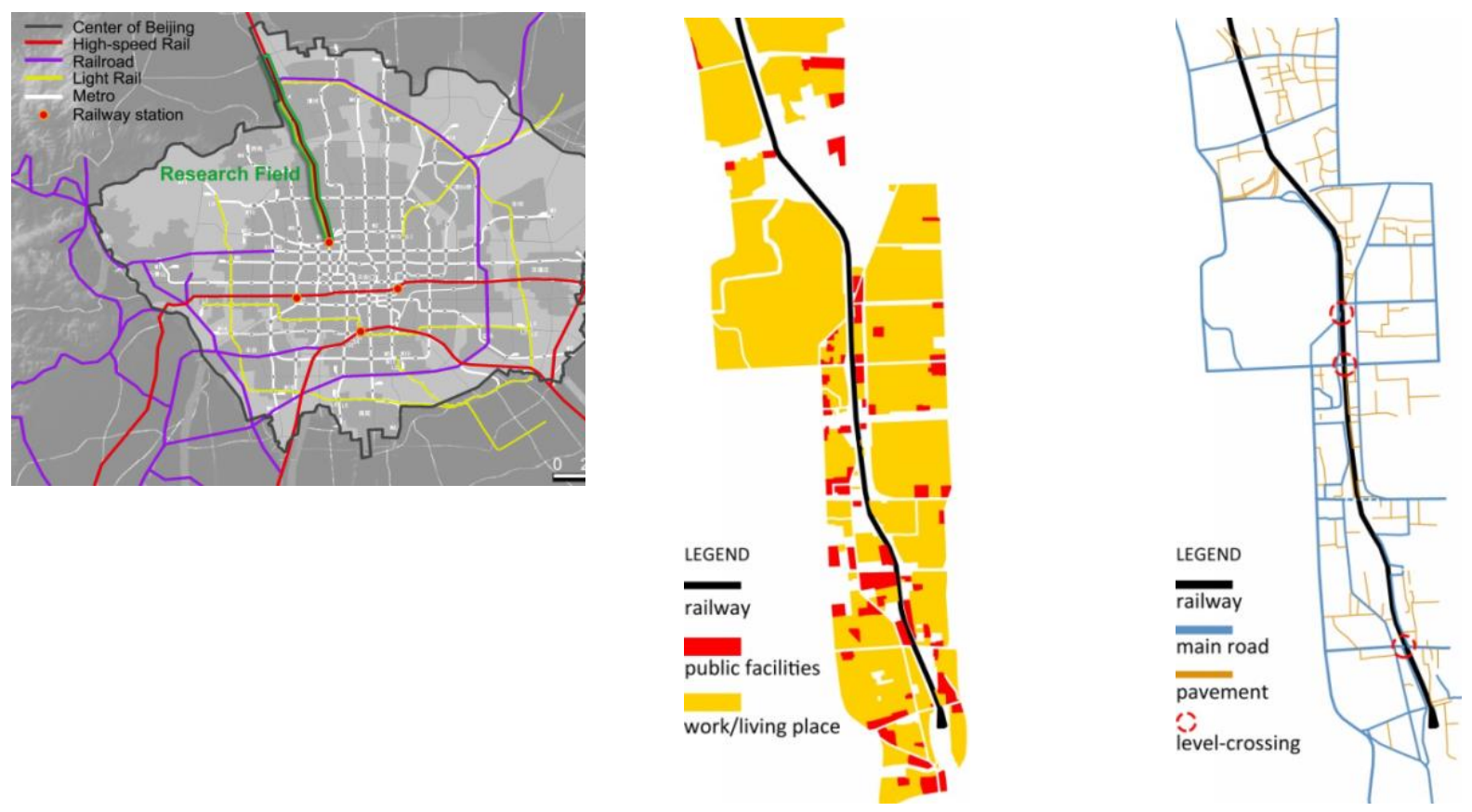

Fig. 3 The position of the research field in

Beijing.

\subsection{Socio-demographic data input}

Fig. 4 The visual (left) and physical (right) disconnect of the urban areas around the Beijing-Zhangjiakou railway. 
The 6th population census of Beijing (Department of Population and Employment Statistics National Bureau of Statistics 2010), which provides demographic distribution at the district level, is used to generate the agent population. Since our case study is located in Haidian district, the number of density (people/ha2) is assigned to residential areas by taking into account the statistics of permanent residents and the ratio of residential land-use in Haidian district. Furthermore, the synthetic population is categorized by a scale of worker: non-worker: visitor, which is 5:4:1 in this case. The number of households according to household (hh) size in Haidian district (2.46 people/hh) is also considered. Finally, vehicles are generated in each household with a number of $0.6 \mathrm{car} / \mathrm{hh}$.

\subsection{Geographical data input}

In order to study the potential effects of various street networks on walking and driving behaviors, the area of an $800 \mathrm{~m}$ radius around the railway track with a buffer of $2500 \mathrm{~m}-3000 \mathrm{~m}$ away from the railway is considered as the case study area. The five types of activities are located on the land-use layer based on two open source maps: the parcel map for Beijing (Long and Liu 2013) and the POIs (Points of Interest) map of (China-latest-free 2017). In addition, two types of road networks are loaded into the model with nearly 7000 road segments. In order to count the total on-road pollutants, an emission standard for cars (Category $\mathrm{M}^{*}$ ) is given to vehicles, which is $80 \mathrm{~g} / \mathrm{m} \mathrm{NO}_{2}$ (Beijing introduced the Euro IV standard and most of the Chinese private cars consume petrol).

\subsection{Activity pattern input}

The final input is activity pattern created from the 2008 Time Use Survey (TUS) in China (Department of Social and Science and Technology Statistics National Bureau of Statistics 2008), which was carried out for relevant policy-making and reflecting the Chinese lifestyle. Results in this survey represent seven kinds of human activities on weekdays and weekends, with differences between male and female, urban and rural. Based on this data, an activity schedule is developed for the urban area in Beijing on a typical weekday (see Fig. 5) and a weekend (see Fig.6).

\subsection{Results}


After initialization, agents depart from homes to workplaces and public spaces by taking the shortest routes. The total number of car agents drove on each road segment is output hourly by the model. For many main roads have more than two parallel lanes, the traffic on these lanes are summed up by using a Python algorithm. As a validation, we compare our result with the real-time on-road traffic data from Google maps. Fig. 7 shows an example of the traffic volume within the research buffer, $2500 \mathrm{~m}-3000 \mathrm{~m}$ away from the railway, at 10:00 AM on a weekday. Had calculated by COPERT, the traffic volume is then transformed to emission rates $(\mu \mathrm{g} /(\mathrm{s} * \mathrm{~m}))$. Afterwards, the hourly emission rates are assigned to each road segment and visualized in heat maps. Fig. 8 describes the distribution of $\mathrm{NO}_{2}$ emission in a neighborhood scale, $800 \mathrm{~m}$ away from the railway at the same time of Fig. 7. For the next step, different urban design scenarios could be simulated and compared by means of changing the input spatial data (e.g. the connectivity of road network and public spaces distribution).

\section{Discussions and conclusion}

This paper explored the relationship between the transport infrastructure, human behavior and ecosystem, resulting in a conceptual model of the urban infrastructure development inspired by the double helix structure of DNA. Based on this model, an integrated human-needs driven urban design and computational evaluation methodology with supports for decision-making process was proposed. Since policy-making is an interconnected network involving distinct stakeholders, this paper introduced a transparent agent-based model for the evaluation of the impacts of an integrated urban transport infrastructure and public space design on human behavior and environmental quality. Finally, the evaluation part of the integrated methodology was applied in a case study in Beijing to simulate the effects of a baseline transport-public space design scenario on local air quality.

Future work will focus on testing different design scenarios and evaluating their impacts on human behavior and air quality. This could eventually lead to a comparison of different integrated transport-public space plans for supporting the policy-making process. It is expected that our methods can be applied to a wide range of transport design and evaluation applications to satisfy both human and ecology needs, and to involve civic participatory into the policy-making process. 

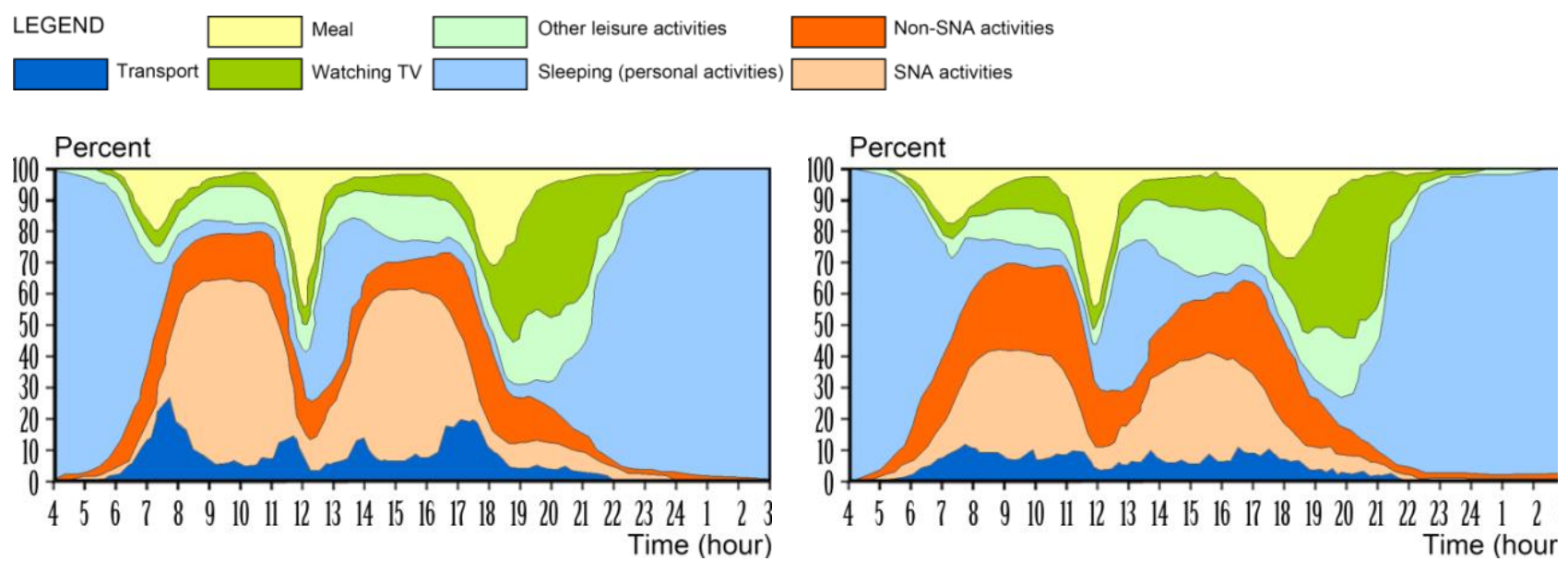

Fig. 5 The chart of activity pattern on weekdays (adapted

from the 2008 Time Use Survey).

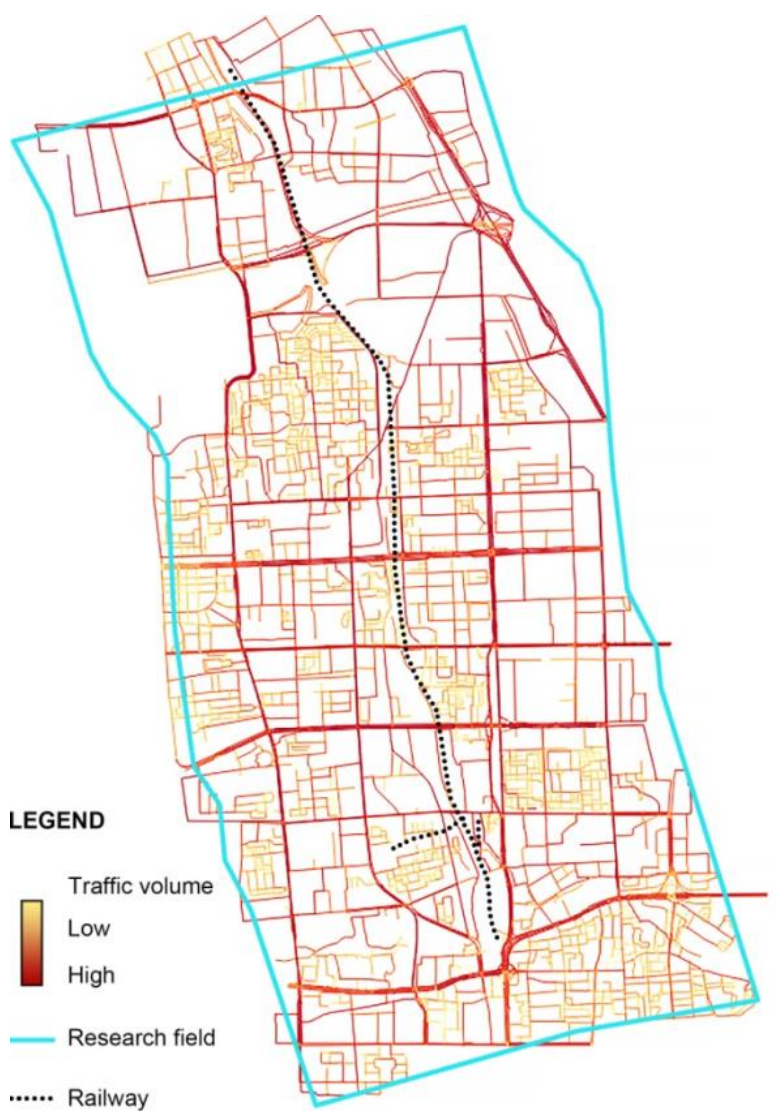

Fig. 7 Simulation result of the traffic volume within the

buffer of 2500m-3000m away from the railway, 10:00 AM

on a weekday.
Fig. 6 The chart of activity pattern on weekends (adapted

from the 2008 Time Use Survey).

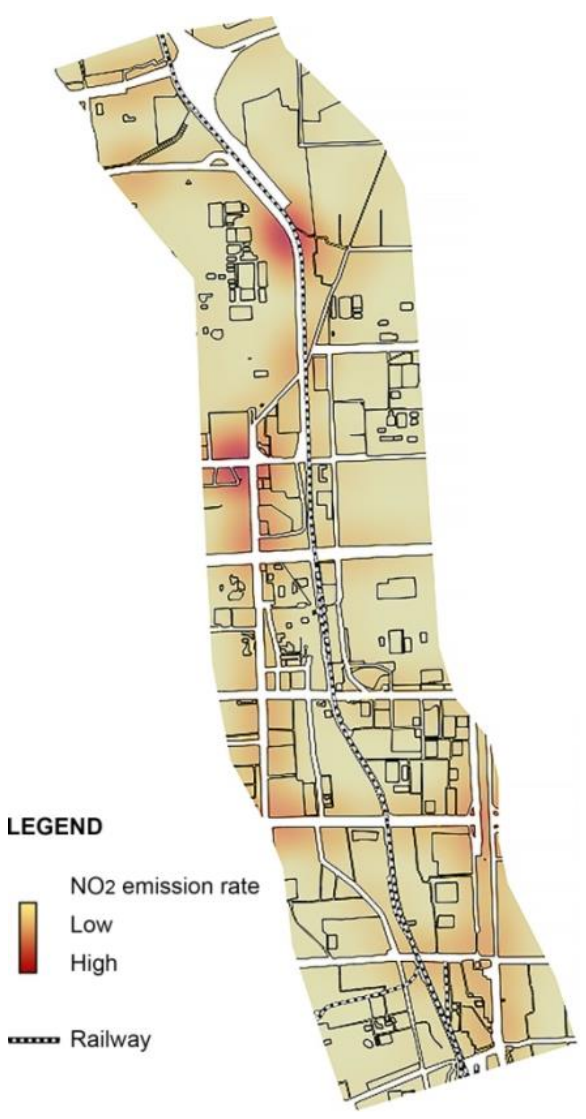

Fig. 8 Visualization of the $\mathrm{NO}_{2}$ pollution within the area of an $800 \mathrm{~m}$ radius around the railway, 10:00 AM on a weekday. 


\section{References}

Alberti, M., \& Marzluff, J. M. (2004). Ecological resilience in urban ecosystems: Linking urban patterns to human and ecological functions. Urban Ecosystems, 7(3), 241-265, doi:10.1023/B:UECO.0000044038.90173.c6.

Axelrod, R. M. (1976). Structure of decision: The cognitive maps of political elites. Princeton, US: Princeton university press.

Axelrod, R. M. (1997). The complexity of cooperation: Agent-based models of competition and collaboration: Princeton University Press.

Batty, M., Axhausen, K. W., Giannotti, F., Pozdnoukhov, A., Bazzani, A., Wachowicz, M., et al. (2012). Smart cities of the future. [Article]. European Physical Journal-Special Topics, 214(1), 481-518, doi:10.1140/epjst/e2012-01703-3.

Bustos-Turu, G., Acha, S., Van Dam, K., \& Shah, N. (under review). Electric vehicle smart charging strategies in urban energy systems: An integrated agent-based and multi-objective modelling approach. currently under review for Applied Energy Journal.

Carmona, M. (2003). Public places, urban spaces: the dimensions of urban design. Oxford: Architectural Press.

China-latest-free (2017). Geofabrik GmbH. https://download.geofabrik.de/asia/china.html. Accessed 2018-02-28.

De Nazelle, A., Nieuwenhuijsen, M. J., Antó, J. M., Brauer, M., Briggs, D., Braun-Fahrlander, C., et al. (2011). Improving health through policies that promote active travel: a review of evidence to support integrated health impact assessment. Environment international, 37(4), 766-777.

De Nazelle, A., Rodríguez, D. A., \& Crawford-Brown, D. (2009). The built environment and health: impacts of pedestrian-friendly designs on air pollution exposure. Science of the Total Environment, 407(8), 2525-2535.

Department of Population and Employment Statistics National Bureau of Statistics (2010). Tabulation on the 2010 Population Census of the People's Republic of China. China: China Statistics Press and Beijing Info Press.

Department of Social and Science and Technology Statistics National Bureau of Statistics (2008). 2008 Time Use Survey in China. China: China Statistics Press.

Forrester, J. W. (1969). Urban Dynamics. Cambridge, US: MIT Press. 
Gan, W. (2014). Responsive urban simulation: An approach towards real time evaluation of urban design projects. Master's Thesis, Politecnico Di Milano, Milano, Italy.

Geddes, S. P. (1915). Cities in evolution: an introduction to the town planning movement and to the study of civics. London, UK: Williams \& Norgate London.

Horni, A., Nagel, K., \& Axhausen, K. W. (2016). The multi-agent transport simulation MATSim. UK: Ubiquity Press London.

Jackson, T., Jager, W., \& Stagl, S. (2004). Beyond insatiability: needs theory, consumption and sustainability. ESRC Sustainable Technologies Programme Working Paper Series, 2.

Long, Y., \& Liu, X. (2013). Automated identification and characterization of parcels (AICP) with OpenStreetMap and Points of Interest. Beijing City Lab, Working paper \# 16.

Mallmann, C. (1980). Society, needs and rights: a systemic approach. Human Needs: A contribution to the current debate, $37-54$.

Ravazzoli, E., \& Torricelli, G. P. (2017). Urban mobility and public space. A challenge for the sustainable liveable city of the future. The Journal of Public Space, 2(2), 37-50.

Skiena, S. S. (1998). The Algorithm Design Manual: Springer Science \& Business Media.

UN-Habitat (2015). Global Public Space Toolkit: From Global Principles to Local Policies and Practice. Nairobi: United Nations Human Settlements Programme.

van Dam, K. H., Bustos-Turu, G., \& Shah, N. A methodology for simulating synthetic populations for the analysis of socio-technical infrastructures. In J. e. al (Ed.), Advances in Social Simulation 2015, 2017 (pp. 528): Springer

van Dam, K. H., Koering, D., Bustos-Turu, G., \& Jones, H. (2014). BOTH Agent-based simulation as an urban design tool-Iterative evaluation of a smart city masterplan.

Varnelis, K. (2008). The infrastructural city: Networked ecologies in Los Angeles. Barcelona: Actar.

Zellner, M. L. (2008). Embracing Complexity and Uncertainty: The Potential of Agent-Based Modeling for Environmental Planning and Policy. Planning Theory \& Practice, 9(4), 437-457, doi:10.1080/14649350802481470. 
Zhao, P., Chapman, R., Randal, E., \& Howden-Chapman, P. (2013). Understanding Resilient Urban Futures: A Systemic Modelling Approach. Sustainability, 5(7), 3202-3223, doi:10.3390/su5073202. 